\title{
Breeding for Low Temperature Stress Tolerance in Reproductive Stage of Rice (Oryza sativa L.)
}

\author{
Bapsila Loitongbam*, Kailash Chandra, Prashant Bisen, Namrata, Padma Thakur and Sandhya
}

Dept. of Genetics and Plant Breeding, Institute of Agricultural Sciences, Banaras Hindu University, Varanasi (221 005), India

\section{Corresponding Author}

Bapsila Loitongbam

e-mail: bapsi90@gmail.com
Article History

Article ID: 3 C0829

Received in $7^{\text {th }}$ November, 2017

Received in revised form $29^{\text {th }}$ November, 2017

Accepted in final form $7^{\text {th }}$ December, 2017

\begin{abstract}
Rice is a cold-sensitive plant that has its origin in tropical or subtropical areas and cold damage can cause serious yield losses. Low temperature affects the rice cultivation mainly in two stages of development i.e. seedling and booting. In both of them, cold temperature has harmful effects on crop productivity. Low temperature during the reproductive stage in rice causes degeneration of spikelets, incomplete panicle exsertion and increases spikelet sterility thus reducing grain yield. The most sensitive stage to low temperature is the booting stage. The percentage of fertile spikelet has been used as effective parameters of cold tolerance of rice at booting stage. Low temperature at booting stage causes anther injury, degeneration of young microspores, resulting in high spikelet sterility and reduced rice yield. Understanding molecular mechanism of cold stress in rice became an important step since it will increase the precision in screening in addition to phenotypic evaluation. Cold tolerance in rice is a quantitative trait controlled by multiple genes. Because it is often difficult to directly associate plant phenotypes with the genes responsible for cold tolerance, therefore marker-assisted selection is an effective means of developing coldtolerant cultivars. Dissecting cold stress-mediated physiological changes and understanding their genetic causes will facilitate the breeding of rice for cold tolerance. Research on quantitative trait loci (QTL) related to cold stress at the germination, seedling and reproductive stages that will provide useful information to accelerate progress in breeding programme.
\end{abstract}

Keywords: Cold Stress, Booting, Seedling, Spikelet Fertility, Yield

\section{Introduction}

Rice (Oryza sativa L.) is the second most important staple food crop in the world and has originated in a tropical region and 90 per cent of the rice is produced and consumed in South East Asia. More than half of the world population depends upon rice as it is the staple food of many developed and developing countries around the globe. The physiological metabolisms of rice are negatively affected by various abiotic stresses by altering its grain yield. Cold stress is one of the abiotic stresses which are common problem of rice cultivation. It causes various injuries to rice seedlings in low-temperature and high-altitude areas and is therefore an important factor affecting rice production in such areas (Xiao et al., 2015). Rice is a cold-sensitive plant that has its origin in tropical or subtropical areas, and cold damage can cause serious yield losses. Low temperature effects the rice cultivation mainly in two stages of development i.e. seedling and booting. In both of them, cold temperature has harmful effects on crop productivity, as in the first case the number of established plants is affected and in the booting stage pollen sterility can be induced by cold, decreasing the final number of grains. Apart from the two critical stages, low temperature stress can also be manifested at different growth stages such as germination, seedling, vegetative, reproductive, and grain maturity (Andaya and Mackill, 2003a). Cold stress has many negative impacts on the productivity of rice cultivars like it reduces growth of seedling, weakens photosynthetic ability, reduces plant height, delays days to heading, reduces spikelet fertility, and cause poor grain quality (Suh et al., 2010). It may also cause various seedling injuries, delayed heading and yield reduction due to spikelet sterility. The seedlings treated under low temperature suffer severe stunting, leaf yellowing and also appear necrotic as brown spots on the stem (Andaya and Mackill, 2003b). Therefore, cold tolerance at the early seedling stage is of particular importance, especially to direct seeded rice cultivation. Low temperature tolerance at the seedling stage has also been evaluated by determining survival percentages, as susceptible seedlings have problems in maintaining normal metabolic rates under cold and ultimately die (Morsy et al., 2007). The most sensitive stage to low temperature is the booting stage. The percentage of fertile spikelet has been used as effective parameters of cold tolerance of rice at booting stage. Low temperature at booting stage causes anther injury, degeneration of young microspores, resulting in high spikelet sterility and reduced 
rice yield. Cold temperature during the reproductive phase leads to seed sterility, which reduces yield and the grain quality of rice. The fertilization stage, ranging from pollen maturation to the completion of fertilization, is sensitive to unsuitable temperature. In cold temperature areas, improving cold tolerance at the fertilization stage (CTF) is an important objective of rice breeding program. Low temperature at the booting stage has also been reported to cause degeneration of young microspores, and hypertrophy and dissolution of tapetal cells, interrupting or decreasing the supply of nutrients from the anther walls to the pollens (Satake, 1989). Crops are exposed to varied environmental conditions during their life cycle. Cold stress is a major environmental factor limiting the growth, productivity, and geographical distribution of crops. It can be classified as chilling $\left(0-15^{\circ} \mathrm{C}\right)$ and freezing $\left(<0{ }^{\circ} \mathrm{C}\right)$ stress. As rice is originated in tropical and subtropical regions, it is more sensitive to cold stress than other cereal crops such as wheat (Triticum aestivum L.) and barley (Hordeum vulgare L.). Therefore, in this review we focus on the low temperature stress mainly in the reproductive stage of rice that can adversely affect grain quality or cause yield reductions and also their molecular intervention.

\section{Effects of Cold Stress in Rice}

Even though cold temperature affects rice growth from seed germination to seed maturity, cold temperature at reproductive phase decrease seed set. Two stages of the reproductive phase in rice are known to be the most sensitive to cold temperature: booting stage and fertilization stage. At booting stage, ranging from pre-meiotic mother cells to microspores and pollen maturation, cold temperature can disrupt mitosis I and II, which prevents rice microspores from maturing into normal tricellular pollen grains (Satake, 1989). The fertilization stage begins just after pollen maturation and the complete fertilization stage consists of anther dehiscence, pollen germination, pollen tube elongation and fertilization. Due to the negative effects of low temperature on rice growth, cold tolerance is an important feature for both, temperate and high altitude, regions. Ghadirnezhad and Fallah (2014) reported that low temperature had significant effect on all characters, such as number of panicles, length of panicle, and number of full, empty, and total grains; as a result, yield was significantly reduced. Further, they discovered the interaction between temperature and varieties showed that most tolerant variety in relation to temperature stress had least percentage yield decrease (19\%) was shirudi variety and the most sensitive one with most percentage of yield decrease (29\%) was local tarom variety. Xu et al. (2008) evaluated cold tolerances of $F_{2}$ plants on the basis of spikelet fertility of main panicles at seed ripening stage, and those of $F_{3}$ lines were evaluated as mean spikelet fertility of the main panicles from 15 to 20 plants in each line. They reported severe reduction of spikelet fertility in susceptible lines. Many species of tropical or subtropical origin are injured or killed by nonfreezing low temperatures, and exhibit various symptoms of chilling injury such as chlorosis, necrosis, or growth retardation. In contrast, chilling-tolerant species are able to grow at such low temperatures (Sanghera et al., 2011).Ye et al. (2009) reported that yield losses due to cold temperature are a result of incomplete pollen formation and subsequent floret sterility. In Australia, rice farmers suffered losses ranging from 0.5 to $2.5 \mathrm{t} / \mathrm{ha}$ in $75 \%$ of the years due to low temperature during the reproductive stage. During the grain filling stage, chilling temperature may cause delayed and incomplete grain maturation. Transferring cold tolerance from different sources to locally adapted cultivars requires the presence of the selective agent, in this case the low temperature. However, its abiotic nature makes it unpredictable under field conditions in terms of its intensity, duration, and timing that limit field selection for cold tolerance in rice (da Cruz and Milach, 2000). Singh et al. (2005) identified overseas rice varieties that were cold tolerant under local weather conditions and by using those genotypes as parent material, developed cold tolerance varieties of rice. Kim et al. (2011) identified two SNPs (single nucleotide polymorphisms) in OsGSTZ2 and suggested that cold sensitivity in rice is strongly correlated with a naturally occurring Ile99Val mutation in the multifunctional glutathione transferase isoenzyme GSTZ2. Parveen et al. (2013) observed the effect of low temperature stress on two rice varieties (Basmati-385 and Shaheen). They found the concentrations of photosynthetic pigments were negatively affected by low temperature stress in both varieties. In Basmati-385, chlorophyll 'a' content had a maximum value $(5.731 \mathrm{ug} / \mathrm{g}$ ) at $-2{ }^{\circ} \mathrm{C}$ and the lowest value $(4.64 \mathrm{ug} / \mathrm{g})$ was seen at -4 ${ }^{\circ} \mathrm{C}$. Chlorophyll ' $\mathrm{b}$ ' content was also decreased under low temperature stress in Basmati-385, showing the lowest value $(4.1 \mathrm{ug} / \mathrm{g})$ at $0^{\circ} \mathrm{C}$. Suzuki et al. (2008) reported that the effects of cold stress at the reproductive stage of plants delay heading and result in pollen sterility, which is thought to be one of the key factors responsible for the reduction in grain yield of crops.

\section{Physiological Basis of Cold Tolerance at Booting Stage}

Most of the physiological analyses to study the cold tolerance or sensibility of rice have been made in two stages of development: seedling and booting. In both the stages cold temperature has harmful effects on crop productivity, but booting stage causes more harm by decreasing grain yield. Zhou et al. (2010) conducted an evaluation of cold tolerance of ZLI929 and Towada and found that the spikelet fertility was an appropriate index to evaluate cold tolerance at the booting stage. Cold tolerance at the booting stage (CTB) has been improved in rice breeding program. CTB is evaluated by seed fertility from pre-meiotic mother cells until grain maturity in the field using cold-water irrigation at $18-19^{\circ} \mathrm{C}$. And they further evaluated cold tolerances by the spikelet fertility of the main panicles at the seed ripening stage, and found most of the recombinant $F_{2}$ plants were heterozygous. Cold tolerances of recombinants were evaluated as mean spikelet fertilities of 
the main panicles from 8 to 10 selected homozygous plants in each family. They also evaluated and compared pollen fertility for cold tolerance related traits for ZL1929, a $\mathrm{BC}_{6} \mathrm{~F}_{4}$ coldtolerant near-isogenic lines (NILs) developed by backcrossing KMXBG as donor to the cold-sensitive Japanese commercial japonica cv. Towada, and selecting cold-tolerant individuals in each generation of backcrossing. Zhou et al. (2012) developed cold tolerant near-isogenic lines (NILs) by backcrossing Kunmingxiaobaigu (KMXBG), reported to be the most coldtolerant variety at the booting stage, with the cold sensitive Japanese commercial japonica variety, Towada. Shinada et al. (2013) selected Eikei88223 and Hoshinoyume as vigorous CTF (cold tolerant at flowering) cultivars, and Suisei as a very weak CTF cultivar to characterize morphological changes (including pollen maturity, pollen germination on the stigma and pollen tube elongation in the style) during the fertilization stage by the cold temperature treatment. And they investigated the influence of the cold temperature treatment on pollen maturity at the fertilization stage with $\mathrm{I}_{2} \mathrm{KI}$ staining and further they observed several stages of pollen grains in the anthers from spikelets before flowering at 3, 7, or 10 days after the start of the cold temperature treatment. Pollen grains staining black were considered as mature, while those staining red as immature or degraded. In this type of pollen grain, starch around the whole of the cell wall was degraded. Pollen grains staining red with an abnormal shape were defined as seriously degraded. Pollen grains without staining and a normal shape were defined as immature. da Cruz et al. (2006) investigated the effect of cold temperatures in six rice genotypes at $17^{\circ} \mathrm{C}$ for varying length of time (three, five, seven and ten days) at two reproductive stages (microsporogenesis and anthesis). They measured cold tolerance as the percentage of reduction in panicle exsertion and in spikelet fertility. Shirasawa et al. (2012) identified a quantitative trait locus (QTL) for cold tolerance on the long arm of chromosome 3 and they used seed fertility, as an index of cold tolerance which is the percentage of the number of fertile seeds in the number of florets, of plants grown under low temperature stress. Chhun et al. (2007) conducted phenotypic analysis on rice GA-related mutants, including GA-deficient and GA-insensitive mutants. They found that the GA-deficient mutant reduced pollen elongation 1 is defective in pollen tube elongation, resulting in a low fertilization frequency, whereas the GA-insensitive semi dominant mutant SIr1-d3 is mainly defective in viable pollen production. Gene expression analysis has contributed to increase our knowledge about the physiological mechanisms related to abiotic stresses in plants, including low temperature tolerance (Gao et al., 2008). Besides a precise selection method, the genetic basis of cold tolerance also affects the breeding for this trait. Imin et al. (2004) extracted proteins at the trinucleate stage, and separated by two dimensional gel electrophoresis (2-DE) at the young microspore stage and compared for four days at $12{ }^{\circ} \mathrm{C}$. More than 3000 rice anther proteins of cold- sensitive cultivar Doongara plants at the trinucleate stage were resolved on 2-DE gels over a $\mathrm{pH}$ range of 4-7 and detected by silver-staining. Further they observed different types of abnormal anthers in cold treated samples including irregular shaped small, white or light yellowish anthers. Jiang et al. (2010) in their study used RIL population consisting of 151 lines, which was developed by single seed descent (SSD) method. They conducted screening of the chilling tolerance in various environments including a cold water treatment in Chuncheon, a naturally low temperature high altitude environment in Kunming and a naturally low temperature high latitude environment in Yanji and performed two years of experiment. They induced chilling injury in three low temperature treatment locations and study the responses against different low temperature environment. Their results showed that many $G X E$ interactions of chilling related traits were correlated with temperature factors such as daily average, minimum, maximum and diurnal temperature range. Oliver et al. (2005) used the Australian cultivar Doongara and the Chinese cultivar R31 as models of a cold-sensitive and a cold tolerant cultivar, respectively. In their experiment they showed that the time of highest sensitivity to cold coincides with the time of peak tapetal activity: the transition of the tetrad to early uninucleate stage (young microspore, YM stage). Further, they found that the low temperatures at this stage of pollen development result in an accumulation of sucrose in the anthers, accompanied by decreased activity of cell wall bound acid invertase and depletion of starch in mature pollen grains which leads pollen sterility and decreased grain yield. Saito et al. (2010) mapped Ctb1 to a 17-kb region containing two genes that encode an F-box protein and a ser/thr protein kinase. They identified that the F-box protein gene was preferentially expressed in young panicles, while the ser/thr protein kinase gene was expressed in leaves and young panicles. Further, they assessed the cold tolerance of $T_{2}$ and $T_{3}$ progenies by measuring the degree of spikelet fertility in plants treated with cool water irrigation (19 ${ }^{\circ} \mathrm{C}, 25 \mathrm{~cm}$ ) or cool air $\left(12^{\circ} \mathrm{C}, 4\right.$ days). They identified the F-box protein gene confers cold tolerance and also cold tolerance is associated with greater anther length, and the transgenic plants had longer anther anthers. Pandey and Gupta (1993) used the intercrossed of four cold-tolerant rice varieties, viz. 'Khonorulo', 'Namyi', 'Abor B' and 'Meghalaya-l' and with two cold-susceptible ones, viz. 'Pusa 33' and 'Subhadra' (DR92), to study the inheritance of genes governing panicle exsertion and their allelic relationship among cold tolerant varieties. They found the complete panicle exsertion of $F_{1}$ hybrid indicating dominance of this trait but the absence of segregation for panicle exsertion of $F_{2}$ and backcrosses show that that the dominant genes in all the four cultivars are allelic.

\section{Molecular Basis of Cold Tolerance}

Understanding molecular mechanism of cold stress in rice became an important step since it will increase the precision in screening in addition to phenotypic evaluation. Large numbers 
of studies have been done to improve the cold tolerance; few of them are illustrated below. Zhou et al. (2010) conducted an experiment by using a total of 647 simple sequence repeat (SSR) markers distributed across 12 chromosomes to survey for polymorphisms between ZL1929-4 and the cold-sensitive Japonica cultivar Towada. They detected and mapped a QTL for cold tolerance with major effect at the booting stage on the long arm of chromosome 7 and further establish functional roles for genes involved in cold tolerance by fine mapping and cloning of cold-tolerance genes and ultimately use those genes in breeding modern cold tolerant rice varieties. Genetic map has been constructed by using 191 recombinant inbred lines derived from a cross between a temperate japonica, M-202, and a tropical indica, IR50, in order to locate quantitative trait loci (QTLS) conferring cold tolerance. And they detected the QTLs controlling booting- stage-cold tolerance were not associated with seedling cold tolerance Andaya and Mackill (2003b). Suh et al. (2010) in their study identified and analyzed QTLs derived from a new genetic source (IR66160-121-4- 4-2) by using an $\mathrm{F}_{7-8}$ RILs through phenotyping in the field with cold-water irrigation as well as with cool air temperature in the greenhouse with respect to spikelet fertility trait (percent seed set). They reported the identification of three reliable QTLs located on chromosomes 3, 7, and 9 responsible for cold tolerance, and haplotype analysis of QTL alleles and their validation using linked SSR marker analysis on some representative cold-tolerant RIL genotypes. Kuroki et al. (2007) analyzed a quantitative trait locus (QTL) for cold tolerance at booting stage of cold-tolerant rice breeding line, Hokkai-PL9. In their study a total of 487 simple sequence repeat (SSR) markers were distributed throughout the genome to survey for polymorphism between Hokkai-PL9 and a cold sensitive breeding line, Hokkai 287 and 54 markers were polymorphic. Further by conducting interval mapping using an $\mathrm{F}_{2}$ population between Hokkai-PL9 and Hokkai287, a QTL for cold tolerance was detected on the short arm of chromosome 9. Shinada et al. (2013) conducted QTL analysis for CTF using 120 backcrossed inbred lines derived from a cross between Eikei88223 (vigorous CTF) and Suisei (very weak CTF). They identified three QTLs for CTF and a clear effect by QTL, qCTF7, for increasing the level of CTF was validated using advanced progeny. Shinada et al., (2014) evaluated the effect of pyramiding QTLs for improvement of CTF level using eight possible genotype classes from the $152 \mathrm{~F}_{3}$ population derived from a cross between Eikei88223 and Suisei. And they detected the increasing of CTF levels in combinations between qCTF7 and qCTF12 and between qCTF8 and qCTF12. Furthermore, they compared the haplotype pattern around the QTLs for CTF among the rice cultivars from Hokkaido.

The molecular characterization of novel cold-tolerant strains derived from crosses with Silewah was performed to identify quantitative trait loci (QTLs) responsible for cold tolerance. Molecular marker analysis revealed that 2 cold-tolerant strains carried chromosomal segments of Silewah at the same genomic regions on chromosomes 3, 4 and 11 . Single marker analysis in segregating population confirmed that the allele of Silewah on chromosome 3 (qCTB3-Silewah) conferred cold tolerance (Mori et al., 2011). Xu et al. (2008) conducted the experiment which involved $1557 \mathrm{BC}_{5} \mathrm{~F}_{2}$ plants and their $\mathrm{F}_{3}$ progenies grown over 2 years and they detected the eight markers which were distributed on chromosomes $1,4,5,10$ and 11 that were associated with cold tolerance. A total of 1,954 RILs composed of $496 F_{10}, 518 F_{6}$ and $940 F_{5}$ individuals, each derived from independent $F_{2}$ plants and advanced through single seed descent, were screened with the microsatellite markers RM5746 and RM3103 which flank qCTS12 and they identified a set of 14 polymorphic marker for refining the qCTS12 locus map position (Andaya and Tai, 2006). Fourteen crosses and backcrosses from five standard rice cultivars, or 21 crosses from seven rice cultivars with different grades of cold tolerance involving nuda, javanica and communis with different grades of cold tolerance were used for the genetic analysis of $F_{1}$ and $F_{2}$ in cold tolerance characters using the method put forward by Griffing II. The gene for cold tolerance at booting stage was identified on chromosome 5 between RM 7452 and RM 31 with genetic distances of 4.8 $\mathrm{cm}$ and $8.0 \mathrm{~cm}$, respectively and this gene explained $10.50 \%$ of phenotypic variance and $5.10 \%$ of phenotypic variance of fully filled grains (Yawen and Xiaoying, 2006). Shirasawa et al. (2012) identified a quantitative trait locus (QTL) for cold tolerance on the long arm of chromosome 3 from the cold tolerant breeding line 'Ukei840' and 'Hitomebore'. They confirmed the effect of this QTL on cold tolerance by developing 'Hitomebore' chromosome segment substitution lines having 'Lijiangxintuanheigu' alleles on chromosome 3. They also found low temperature at the booting stage causes male sterility resulting in severe yield loss. The genetic marker loci/QTL associated with cold tolerance at the booting stage and association mapping were identified in 347 rice accessions with different statistical models. They used 148 SSRs for genotyping and identified 24 markers that were significantly associated with cold tolerance, including five markers in Yunnan and 19 markers in Jilin (Cui et al., 2013). A total of 23 diverse rice cultivars were evaluated for cold tolerance using two screening methods at Chuncheon Substation, National Institute of Crop Science (NICS), Republic of Korea. Three SSR markers were significantly associated with spikelet fertility under cold-water irrigation on chromosomes 1, 2, and 7. Five SSR markers were associated with spikelet fertility under a cool environment greenhouse on chromosomes 8, 9, 10, and 12 . They conclude that SSR marker associated to cold tolerance may also be useful as selection markers in indica/ japonica cross combination to improve cold tolerance (Suh et al., 2013). Cconstructed a linkage map with 135 restriction fragment length polymorphism (RFLP) markers and 34 random amplified polymorphic DNA (RAPD) markers using 212 doubled-haploid lines (DHLs) to identify the chromosomal region controlling cool temperature tolerance (CT) at booting 
stage of rice. They found three QTLs which were mapped to each chromosome: 1, 7 and 11. The QTL with the largest effect, qCT-7, was mapped to chromosome 7 and explained $22.1 \%$ of the total phenotypic variation and the other two QTLs, qCT-1 and qCT-11, explained approximately $5 \%$ each. Further, they also mapped QTLs for heading date and culm length. Kuroki et al. (2009) evaluated cold tolerance at the booting stage on the basis of fertility after cool-water treatment for three years in 114 recombinant inbred lines (RILs) between temperate Japonica Kirara397 (cold sensitive) and Hatsushizuku (cold tolerant). And they detected a QTL for cold tolerance at the booting stage in three trials on chromosome 1 . They also analyzed QTL for heading time and culm length, which are thought to affect cold tolerance and detected every three years on the adjacent region of chromosomes 1 . Ye et al. (2010) identify the QTL controlling low temperature induced spikelet sterility in rice. The QTL (qLTSPKST10.1) was validated and mapped by genotyping the entire $F_{2}$ (282 progeny) and $B C_{1} F_{1}$ (84 progeny) populations. Jena et al., (2010) used $F_{7-8}$ recombinant inbred lines (RILs) which had cold-tolerance genes/quantitative trait loci (QTLs) and selected 15 RILs possessing QTLs for cold tolerance which were phenotype for three main agronomic traits-culm length (CL), days to heading (DTH) and spikelet fertility (SF)-which were most affected during cold stress. The spikelet fertility of the selected breeding lines was much higher (51-81\%) than that of the cold sensitive parent Geumobyeo (7\%) and the selected lines possessed at least one of the three QTLs (qPSST-3, qPSST-7 and qPSST-9) associated with cold tolerance. From these results, it revealed that cold tolerance is associated with spikelet fertility, but independent of the genes controlling culm length and days to heading. Saito et al. (2004) developed Norin-PL8, a cold-tolerant variety of rice by introgressing chromosomal segments from a cold-tolerant tropical japonica variety, Silewah, into a template japonica variety, Hokkai 24. They identified two closely linked quantitative trait loci, Ctb1 and Ctb2, for cold tolerance at the booting stage of Norin-PL8 in the long arm of chromosome 4.

\section{Conclusion}

Cold stress is a serious threat in decreasing the rice yield. Even though some studies have been done in order to understand the cold stress and applying suitable breeding strategy in order to develop cold tolerance. But to combat against severe yield loss and climate change there is need of screening of available genotypes at both phenotypic and genomic level through molecular markers. Identified genomic regions must be incorporate into an elite variety in order to rectify a specific defect.

\section{Reference}

Andaya, V.C., Mackill, D.J., 2003a. Mapping of QTLs associated with cold tolerance during the vegetative stage in rice. Journal of Experimental Botany 54, 2579-2585.
Andaya, V.C., Mackill, D.J., 2003b. QTLs conferring cold tolerance at the booting stage of rice using recombinant inbred lines from a japonica $x$ indica cross. Theoretical and Applied Genetics 106, 1084-1090.

Andaya, V.C., Tai, T.H., 2006. Fine mapping of the qCTS12 locus, a major QTL for seedling cold tolerance in rice. Theoretical and Applied Genetics 113, 467-475.

Chhun, T., Aya, K., Asano, K., Yamamoto, E., Morinaka, Y., Watanabe, M., Kitano, H., Ashikari, M., Matsuoka, M., Ueguchi-Tanakaa, M., 2007. Gibberellin regulates pollen viability and pollen tube growth in rice. The Plant Cell 19, 3876-3888.

Cui, D., Xu, C., Tang, C., Yang, C., Yu, T., Cao, G., Xu, F., Zhang, J., Han, L., 2013. Genetic structure and association mapping of cold tolerance in improved japonica rice germplasm at the booting stage. Euphytica 193, 369-382.

da Cruz, R.P., Milach, S.C.K., 2000. Breeding for cold tolerance in irrigated rice. Ciencia Rural 30, 909-917.

da Cruz, R.P., Milach, S.C.K., Federizzi, L.C., 2006. Rice cold tolerance at the reproductive stage in a controlled environment. Scientia Agricola 63, 255-261.

Gao, J.P., Chao, D.Y., Lin, H.X., 2008. Toward understanding molecular mechanisms ofabiotic stress responses in rice. Rice 1, 36-51.

Ghadirnezhad, R., Fallah, A., 2014. Temperature effect on yield and yield components of different rice cultivars in flowering stage. International journal of Agronomy doi: org/10.1155/2014/846707.

Imin, N., Kerim, T., Rolfe, B.G., Weinman, J.J., 2004. Effect of early cold stress on the maturation of rice anthers. Proteomics 4, 1873-1882.

Jena, K.K., Kim, S.M., Suh, J.P., Kim, Y.G., 2010. Development of cold-tolerant breeding lines using QTL analysis in rice. Proceeding of second Africa Rice Congress on Innovation and Partnerships to Realize Africa's Rice Potential, 22-26, May, 2010, Bamako, Mali.

Jiang, W., Lee, J., Chu, S., Ham, T., Woo, M., Cho, Y., Chin, J., Han, L., Xuan, Y., Yuan, D., Xu, F., Dai, L., Yea, J., Koh, H., 2010. Genotype $\times$ Environment interaction for chilling tolerance of rice recombinant inbred lines under different low temperature environments. Field Crops Research 117, 226-236.

Kim, S.I., Andaya, V.C., Tai, T.H., 2011. Cold sensitivity in rice (Oryza sativa L.) is strongly correlated with a naturally occurring $199 \mathrm{~V}$ mutation in the multifunctional glutathione transferase isoenzyme GSTZ2. Biochemistry Journal 435, 378-380.

Kuroki, M., Saito, K., Matsuba, S., Yokogami, N., Shimizu, H., Ando, I., Sato, Y., 2007. A quantitative trait locus for cold tolerance at the booting stage on rice chromosome 8 . Theoretical and Applied Genetics 115, 593-600.

Kuroki, M., Saito, K., Matsuba, S., Yokogami, N., Shimizu, H., Ando, I., Sato, Y., 2009. Quantitative trait analysis for cold tolerance at the booting stage in a rice cultivar, 
Hatsushizuku. JARQ 43, 115-121.

Mori, M., Onishi, K., Tokizono, Y., Shinada, H., Yoshimura, T., Numao, Y., Miura, H., Sato, T., 2011. Detection of a novel quantitative trait locus for cold tolerance at the booting stage derived from a tropical japonica rice variety Silewah. Breeding Science 61, 61-68.

Morsy, M.R., Jouve, L., Hausman, J.F., Hoffmann, L., Stewart, J.D., 2007. Alteration of oxidative and carbohydrate metabolism under abiotic stress in two rice (Oryza sativa L.) genotypes contrasting chilling tolerance. Journal of Plant Physiology 164, 157-167.

Oliver, S.N., Dongen, J.T.V., Alfred, S.C., Mamun, E.A., Zhao, X., Saini, H.S., Fernandes, S.F., Blanchard, C.L., Sutton, B.G., Geigenberger, P., Dennis, E., Dolferus, R., 2005. Cold-induced repression of the rice anther specific cell wall invertase gene OSINV4 is correlated with sucrose accumulation and pollen sterility. Plant Cell Environment 28, 1534-1551.

Pandey, D.K., Gupta, H.S., 1993. Genetics of panicle exsertion in cold-tolerant rice (Oryza sativa L.). Plant Breeding $111,82-85$.

Perveen, S., Shinwari, K.I., Jan, M., Malook, I., Rehman, S., Khan, M.A., Jamil, M., 2013. Low temperature stress induced changes in biochemical parameters, protein banding pattern and expression of Zat12 and Myb genes in rice seedling. Journal of Stress Physiology and Biochemistry 9, 193-206.

Saito, K., Hayano-Saito, Y., Kuroki, M., Sato, Y., 2010. Map based cloning of the rice cold tolerance gene Ctb1. Plant Science 179, 97-102.

Saito, K., Hayano-Saito, Y., Maruyama-Funatsuki, W., Sato, Y., Kato, Y., 2004. Physical mapping and putative candidate gene identification of a quantitative trait locus $\mathrm{Ctb} 1$ for cold tolerance at the booting stage of rice. Theoretical and Applied Genetics 109, 515-522.

Sanghera, G.S., Wani, S.H., Hussain, W., Singh, N.B., 2011. Engineering cold stress tolerance in crop plants. Current Genomics 12, 30-43.

Satake, T., 1989. Male sterility caused by cooling treatment at the young microspore stage in rice plants, XXIX. The mechanism of enhancement in cool tolerance by raising water temperature before the critical stage. Japanse Journal of Crop Science 8, 240-245.

Shinada, H., Iwata, N., Sato, T., Fujino, K., 2013. Genetical and morphological characterization of cold tolerance at fertilization stage in rice. Breeding Science 63, 197-204.

Shinada, H., Iwata, N., Sato, T., Fujino, K., 2014. QTL pyramiding for improving of cold tolerance at fertilization stage in rice. Breeding Science 63, 483-488.

Shirasawa, S., Endo, T., Nakagomi, k., Yamaguchi, M.,
Nishio, T., 2012. Delimitation of a QTL region controlling cold tolerance at booting stage of a cultivar, 'Lijiangxintuanheigu', in rice, Oriza sativa L. Theoretical and Applied Genetics 124, 937-946.

Singh, R.P., Brennan, J.P., Farrell, T., Williams, R., Reinke, R., Lewin, L., 2005. Economic analysis of breeding for improved cold tolerance in rice in Australia. Australasian Agribusiness 13, 1-9.

Suh, J., Cho, Y., Lee, J., Lee, S., Jung, J., Choi, I., Kim, M., Kim, C., Jena, K. K., 2013. SSR analysis of genetic diversity and cold tolerance in temperate rice germplasm. Plant Breeding and Biotechnology 1, 103-110.

Suh, J.P., Jeung, J.U., Lee, J.I., Choi, Y.H., Yea, J.D., Virk, P.S., Mackill, D.J., Jena, K.K., 2010. Identification and analysis of QTLs controlling cold tolerance at the reproductive stage and validation of effective QTLs in cold-tolerant genotypes of rice (Oryza sativa L.). Theoretical and Applied Genetics 120, 985-995.

Suzuki, K., Nagasuga, K., Okada, M., 2008. The chilling injury induced by high root temperature in the leaves of rice seedlings. Plant Cell Physiology 49, 433-442.

Xiao, N., Huang, W., Zhang, X., Gao, Y., Li, A., Dai, Y., Yu, L., Liu, G., Pan, C., Li, Y., Dai, Z., Chen, J., 2015. Fine mapping of qRC10-2, a quantitative trait locus for cold tolerance of rice roots at seedling and mature stages. Theoretical and Applied Genetics 128, 173-185.

Xu, L., Zhou, L., Zeng, Y., Wang, F., Zhang, H., Shen, S., Li, Z., 2008. Identification and mapping of quantitative trait loci for cold tolerance at the booting stage in a Japonica rice near-isogenic line. Plant Science 174, 340-347.

Yawen, Z., Xiaoying, P., 2006. Genetic analysis for cold tolerance at booting stage for japonica rice (Oryza sativa L.). Indian Journal of Genetics 66, 100-102.

Ye, C., Fukai, S., Godwin, I. D., Koh, H., Reinke, R., Zhou, Y., Lambrides, C., Jiang, W., Snell, P., Redon, E., 2010. A QTL controlling low temperature induced spikelet sterility at booting stage in rice. Euphytica 176, 291-301.

Ye, H., Du, H., Tang, N., Li, X., Xiong, L., 2009. Identification and expression profiling analysis of TIFY family genes involved in stress and phytohormone responses in rice. Plant Molecular Biology 71, 291-305.

Zhou, L., Zeng, Y., Hu, G., Pan, Y., Yang, S., You, A., Zhang, H., $\mathrm{Li}, \mathrm{J}$., Li, Z., 2012. Characterization and identification of cold tolerant near-isogenic lines in rice. Breeding Science 62, 196-201.

Zhou, L., Zeng, Y., Zheng, W., Tang, B., Yang, S., Zhang, H., Li, J., Li, Z., 2010. Fine mapping a QTL qCTB7 for cold tolerance at booting stage on rice chromosome 7 using a near-isogenic line. Theoretical and Applied Genetics 121, 895-905. 